MEDICAL MEMORANDA

\section{Spontaneous Rupture of Intrarenal Arterial Aneurysm}

\section{ROBIN M. JAMESON, AUDREY COUCH}

British Medical fournal, 1972, 3, 28

Spontaneous rupture of a non-calcified intrarenal aneurysm is usually fatal. This case report is of an unusual presentation of this uncommon condition, and the patient remained in good health after treatment.

In a series of 100 non-calcified renal artery aneurysms $25 \%$ ruptured, and $83 \%$ of these ruptured aneurysms were fatal (Ippolito and LeVeen, 1960). Smith and Hinman (1967) stated that $17 \%$ of all renal aneurysms were intrarenal and $20 \%$ of reported intrarenal aneurysms were associated with fatal rupture. Most renal aneurysms are small and asymptomatic. Larger ones may present with renal pain, haematuria, hypertension, and, rarely, a pulsatile renal mass.

\section{Case Report}

The patient, a woman aged 72 , had had seven uncomplicated pregnancies and had reached the menopause at 53 years. She was referred in October 1967 with frequency, backache, and urgency of micturition of six weeks' duration. Two years previously she had had cystitis. Clinical examinaton showed no abnormality apart from a blood pressure of $190 / 110 \mathrm{~mm} \mathrm{Hg}$. The blood urea, haemoglobin, and urine cytological findings were normal. No abnormality was found at cystoscopy. Bacillus proteus was cultured from the midstream urine and was eradicated by antibiotic treatment. The intravenous pyelogram showed a space-occupying lesion in the lower pole of the right kidney (Fig. 1). The left

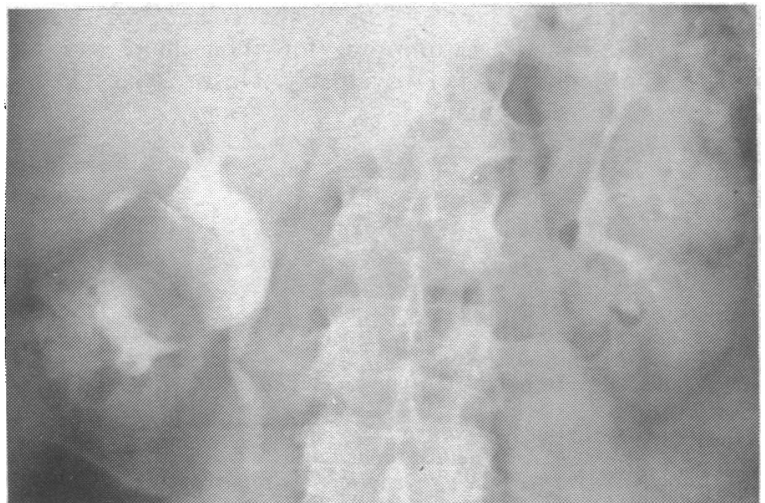

FIG. 1-Intravenous pyelogram showing lesion in lower pole of right kidney.

kidney was normal and there was no vascular calcification. Although malignancy could not be definitely excluded it was thought that the lesion was a simple cyst.

She was symptom-free until nine months later, when she noted an ache in the right loin for two days. Physical examination was negative, the blood pressure was unchanged, no abdominal bruit was heard, and there was no evidence of polyarteritis nodasa. Urine microscopy, culture, and cytological examination showed nothing abnormal.

Regional Urological Centre, Sefton General Hospital, Liverpool 15 ROBIN M. JAMESON, F.R.C.s., Urologist

United Liverpool Hospitals

AUDREY COUCH, D.M.R.D., F.F.R., Radiologist
The full blood count and sedimentation rate were normal but the intravenous pyelogram showed that the lesion in the right kidney had enlarged. Renal angiography was performed by the Seldinger technique. This showed splaying of the renal arteries around the lesion but no abnormal tumour circulation or pooling of the contrast medium (Fig. 2). A vessel inferior to

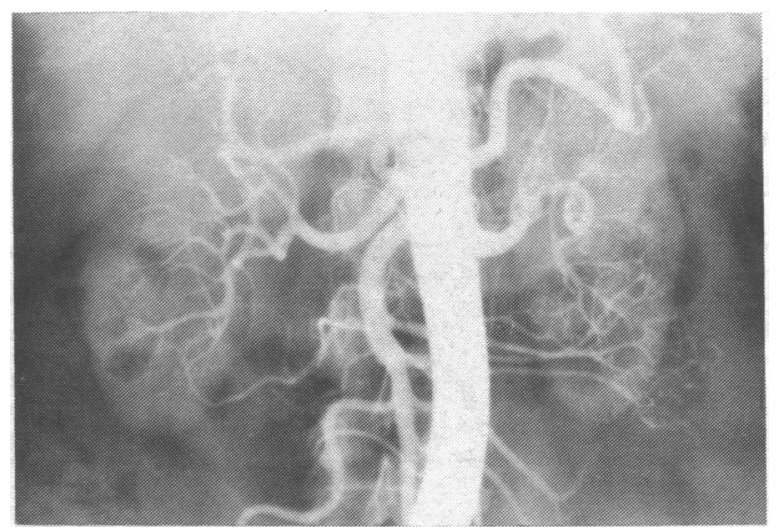

FIG. 2-Intravenous pyelogram showing splaying of renal arteries around lesion.

the lesion was of irregular calibre but the abdominal aorta and its branches were normal, without evidence of generalized arterial disease. The right kidney was explored and found to be of normal size and consistency. Its surface and pedicle appeared normal. It was removed and on section a large blood clot was seen in the middle of the renal substance. There was no evidence of either cyst or tumour. The pathologist reported on the specimen, "there was haemorrhage in the kidney hilum extending outwards to the renal pelvis. This had originated in the rupture of the renal artery" (Fig. 3).

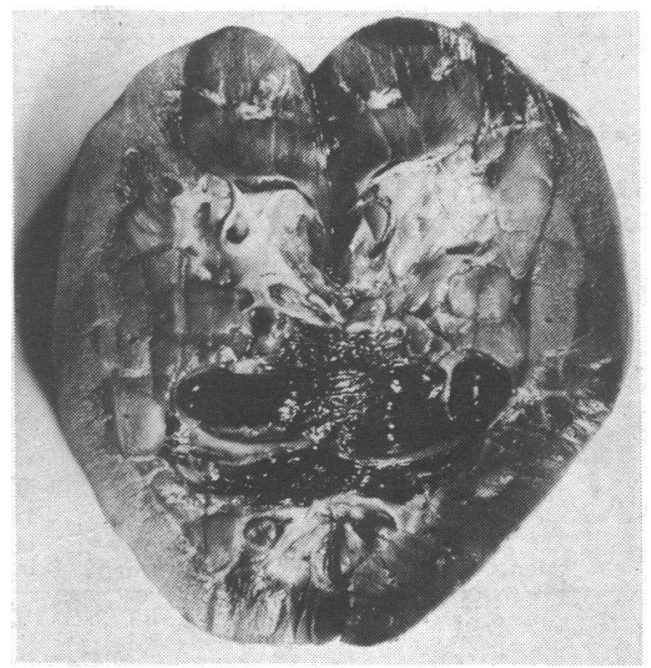

FIG. 3-Section of right kidney containing large blood clot.

She made a straightforward recovery and remained well. At her latest review in September 1971 she was symptom-free, the blood pressure was unchanged, and the blood urea and urine culture were normal.

\section{References}

Ippolito, J. J., and Le Veen, H. H. (1960). Fournal of Urology, 83, 10. Smith, J. N., and Hinman, F. jun. (1967). Fournal of Urology, 97, 990. 